\title{
Foreword to special issue on Rogue Waves
}

\author{
E. Pelinovsky ${ }^{1}\left[\right.$ (D) $\cdot$ K. Belibassakis ${ }^{2} \cdot$ J. Touboul $^{3}$
}

Published online: 9 November 2017

(C) Springer International Publishing AG 2017

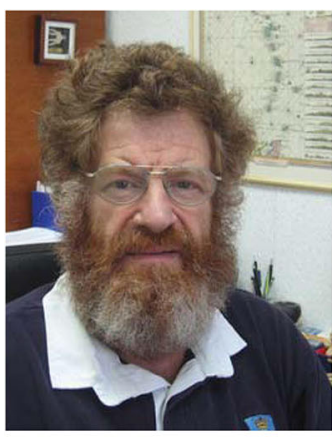

E. Pelinovsky

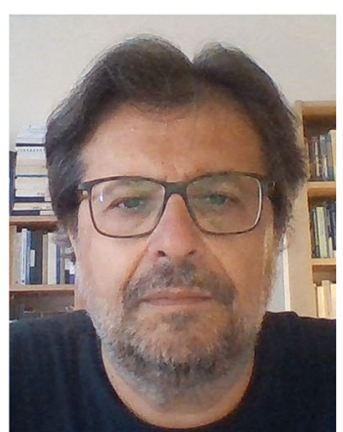

K. Belibassakis

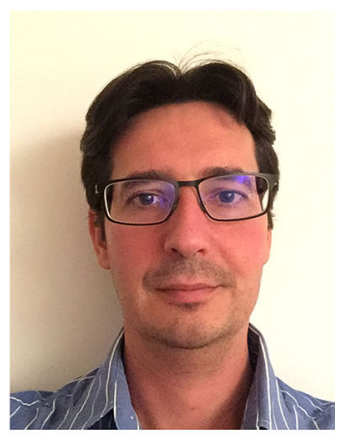

J. Touboul
The terms "freak, rogue, or giant waves" correspond to largeamplitude waves surprisingly appearing on the sea surface ("wave from nowhere", "walls of water", "holes in the sea",

\section{$凶$ J. Touboul}

julien.touboul@mio.osupytheas.fr

https://www.mio.univ-

amu.fr/spip.php?page=pageperso\&id_user $=4923$

E. Pelinovsky

pelinovsky@appl.sci-nnov.ru

http://www.ipfran.ru/english/staff/Pelinovsky.html

K. Belibassakis

kbel@fluid.mech.ntua.gr

http://arion.naval.ntua.gr/ kbel/

1 Institute of Applied Physics and Nizhny Novgorod State Technical University, Nizhny Novgorod, Russia

2 School of Naval Architecture and Marine Engineering, National Technical University of Athens, Zografos 15710, Athens, Greece

3 Université de Toulon, Aix Marseille Université, CNRS, IRD, Mediterranean Institute of Oceanography (MIO), UM 110, La Garde, France and "three sisters"), which can produce serious damages to offshore industry and navigation. Rogue waves have been part of the marine folklore for centuries, but, since the seventies of the last century, oceanographers have started to believe them. Observations gathered by the oil and shipping industries suggest that there really is something like a true monster of the deep that devours ships and sailors without mercy or warning. Several catalogues of rogue-wave observations in the World Ocean are published now demonstrating their reality and wide distribution (Liu 2007; Didenkulova et al. 2006; Nikolkina and Didenkulova 2011, 2012; Baschek and Imai 2011; O'Brien et al. 2017). During the last years, various physical models of the rogue-wave phenomenon have been intensively developed and many laboratory experiments conducted. The main goal of these investigations is to understand the physics of the huge wave appearance and its relation to environmental conditions and to provide the "design" of freak wave needed for engineering purposes. Several books and review papers have been published in the literature (Olagnon and Athanassoulis 2001, 2005, 2009; 
Dysthe et al. 2008; Kharif et al. 2009; Akhmediev and Pelinovsky 2010; Slunyaev et al. 2011; Onorato et al. 2013, 2016).

Now, the study of the freak wave phenomenon is shifted to ocean engineering and the present issue is aimed at showing the progress that was made in the field of rogue wave in the last years. The selection of papers in this issue contains methods based on fully nonlinear potential models, or popular studies based on specific models as Zakharov, NLS, or Witham equations, and results concerning the probability of occurrence of extreme events. In particular, Athanassoulis et al. (2017) studied the effects of sloping bottom and spectral bandwidth on rogue-wave dynamics and kinematics, in finite depth, by means of an exact Hamiltonian coupled-mode system modeling nonlinear refocusing of transient wave groups. The latter are obtained by linear backpropagation of high-amplitude wave trains constructed by the theory of quasi-determinism with application to extreme design waves over variable bathymetry. In the same context, Ducrozet and Gouin (2017), using a nonlinear potential flow solver based on the High-Order Spectral method, examined the influence of varying bathymetry in the occurrence of rogue waves within unidirectional and directional sea-states, focusing on the effect of the directional spreading on the wave statistics. van Groesen and Wijaya (2017) using results from study presented by van Groesen et al. (2017), demonstrated a detection system, based on Xband radars, that could be implemented onboard ships and provide warning up to $60 \mathrm{~s}$ before the event.

Considering unidirectional propagation, Shemer and Chernyshova (2017) studied the nonlinear evolution of narrow-banded wave trains along wave flume both experimentally and numerically using Zakharov equation. The effect of the spectral width on the evolution along the tank is considered for bi-modal initial spectra, and for spectra consisting of a carrier wave and two finite-amplitude sidebands. Good agreement between the experimental results and numerical simulations is obtained. On the other hand, Chabchoub et al. (2017) present results from experimental study describing higher order stage of modulation instability, starting from five-wave systems (main wave frequency and two pairs of unstable sidebands). Laboratory data confirm the NLS wave dynamics forecast by Akhmediev breather solutions. Moreover, Athanassoulis et al. (2017) model rogue waves as localized instabilities emerging from homogeneous and stationary background wavefields under NLS dynamics. The Wigner transform and Penrose's method are used to recover spatially periodic unstable modes, considered as generalized Benjamin-Feir modes, and a criterion for the emergence of rogue waves is formulated. Based on Hamiltonian formalism, Dyachenko et al. (2017) derived a "super compact" equation, amenable to describe nonlinear dynamics of wave group, up to the initial stages of wave breaking. This equation allows definition of exact wave envelop, independently of the spectrum width. Another nonlinear equation was derived by Kharif et al. (2017), who generalized the Whitham equation to take vorticity into account. They considered the behavior of focusing wave groups in the presence of vorticity, and compared their results with fully nonlinear simulations, demonstrating the strong influence of underneath vorticity on extreme wave dynamics.

Furthermore, comparing linear and nonlinear simulations, Chalikov and Bulgakov (2017) have confirmed that probability distributions of water elevation are sensible to nonlinearity, but they observed no effect when considering cumulative probability of wave height, concluding that it could be computed based on results by phase-averaged models, such as WaveWatch III. Finally, Slunyaev and Kokorina (2017) demonstrated that the presence of self-coherent, long-living nonlinear structures that are envelope solitons, whenever present in the wave field, has a strong influence on water waves statistics, making them extreme.

We hope this special issue, presenting some of the latest improvements in understanding rogue-wave formation, from both statistical and deterministic point of view, together with latest developments in rogue-wave prediction will be interesting for specialists in ocean engineering, and help in the development of reliable warning systems.

\section{References}

Akhmediev N, Pelinovsky E. (Eds) (2010) Discussion \& debate: Rogue waves-towards a unifying concept? Europ physical J Spec Topics 185:1-4

Baschek B, Imai J (2011) Rogue wave observations off the US west coast. Oceanography 24:158-165

Didenkulova I, Slunyaev A, Pelinovsky E, Kharif Ch (2006) Freak waves in 2005. Nat Hazards Earth Syst Sci 6(6):1007-1015

Dysthe K, Krogstad HE, Muller P (2008) Oceanic rogue waves. Annu Rev Fluid Mech 40:287-310

Kharif C, Pelinovsky E (2003) Physical mechanisms of the rogue wave phenomenon. Europ J Mech B Fluid 22(6):603-634

Kharif Ch, Pelinovsky E, Slunyaev A (2009) Rogue waves in the ocean. Springer, Berlin, p 216

Liu PC (2007) A chronology of freaque wave encounters. Geofizika 24(1):57-70

Nikolkina I, Didenkulova I (2011) Rogue waves in 2006-2010. Nat Hazards Earth Syst Sci 11(11):2913-2924

Nikolkina I, Didenkulova I (2012) Catalogue of rogue waves reported in media in 2006-2010. Nat Hazards 61(3):989-1006

O’Brien L, Renzi E, Dudley JM, Clancym C, Dias F (2017) Extreme wave events in Ireland: 2012-2016. Nat Hazards Earth Syst Sci Discuss. https://doi.org/10.5194/nhess-2017-206

Olagnon M, Athanassoulis GA (2001) Rogue waves 2000. Actes de Colloques: Proc. Workshop. France: Ifremer p 395

Olagnon M, Athanassoulis GA (2005) Rogue waves 2004. Actes de Colloques: Proc. Workshop. France: Ifremer . p 277

Olagnon M, Athanassoulis GA (2009) Rogue waves 2008. Actes de Colloques: Proc. Workshop. France: Ifremer p 275 
Onorato M, Residori S, Bortolozzo U, Montina A, Arecchi FT (2013) Rogue waves and their generating mechanisms in different physical contexts. Phys Rep 528:47-89

Onorato M, Resitori S, Baronio F (2016) Rogue and Shock Waves in Nonlinear Dispersive Media, Springer, Berlin

Slunyaev A, Didenkulova I, Pelinovsky E (2011) Rogue waters. Contemporary Phys 52(6):571-590

van Groesen E, Turnip P, Kurnia R (2017) High waves in Draupner seas, Part 1: Numerical simulations and characterization of the seas. J Ocean Eng Mar Energy 3(3):233-245

\section{Papers in the present special issue}

Athanassoulis AG, Athanassoulis GA, Sapsis TP (2017) Localized instabilities of the Wigner equation as a model for the emergence of Rogue waves. J Ocean Eng Mar Energy 3(4). https://doi.org/ 10.1007/s40722-017-0095-5

Athanassoulis GA, Belibassakis KA, Papoutsellis CE (2017) An exact Hamiltonian coupled-mode system with application to extreme design waves over variable bathymetry. J Ocean Eng Mar Energy 3(4). https://doi.org/10.1007/s40722-017-0096-4

Chabchoub A, Waseda T, Kibler B, Akhmediev N (2017) Experiments on higher-order and degenerate Akhmediev breather-type rogue water waves. J Ocean Eng Mar Energy 3(4). https://doi.org/10. 1007/s40722-017-0097-3
Chalikov D, Bulgakov KJ (2017) Estimation of wave height probability based on the statistics of significant wave height. J Ocean Eng Mar Energy 3(4):1-7. https://doi.org/10.1007/s40722-017-0093-7

Ducrozet G, Gouin M (2017) Influence of varying bathymetry in rogue wave occurrence within unidirectional and directional seastates. J Ocean Eng Mar Energy 3(4). https://doi.org/10.1007/ s40722-017-0086-6

Dyachenko AI, Kachulin DI, Zakharov VE (2017) Envelope equation for water waves, soliton turbulence and wavebreaking. J Ocean Eng Mar Energy 3(4). https://doi.org/10.1007/s40722-017-0100-z

Kharif C, Abid M, Touboul J (2017) Rogue waves in shallow water in the presence of a vertically sheared current. J Ocean Eng Mar Energy 3(4). https://doi.org/10.1007/s40722-017-0085-7

Shemer L, Chernyshova AJ (2017) Spatial evolution of an initially narrow-banded wave train. J Ocean Eng Mar Energy 3(4). https:// doi.org/10.1007/s40722-017-0094-6

Slunyaev AV, Kokorina AV (2017) Soliton groups as the reason for extreme statistics of unidirectional sea waves. J Ocean Eng Mar Energy 3(4). https://doi.org/10.1007/s40722-017-0099-1

van Groesen E, Wijaya AP (2017) High waves in Draupner seas-Part 2: Observation and prediction from synthetic radar images. J Ocean Eng Mar Energy 3(4). https://doi.org/10.1007/ s40722-017-0090-x 